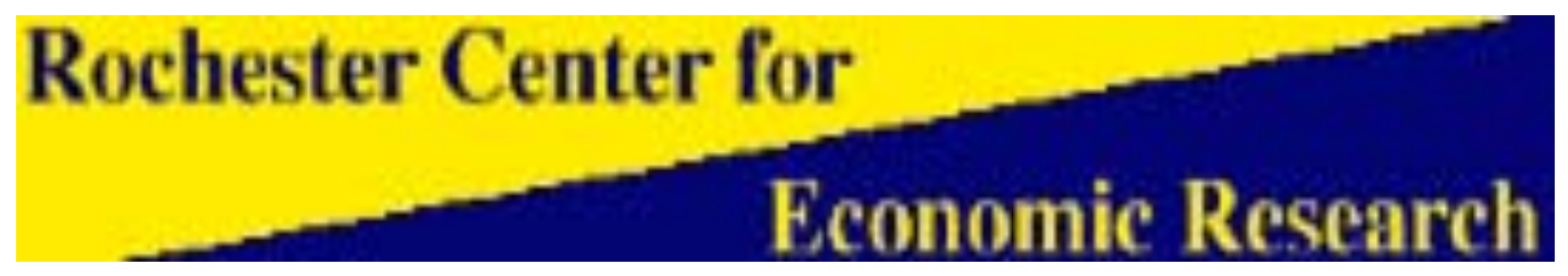

Borrowing-proofness of the Lindahl rule in Kolm triangle economies

Thomson, William

Working Paper No. 555

July 2010 


\title{
Borrowing-proofness of the Lindahl rule in Kolm triangle economies
}

\author{
William Thomson \\ Department of Economics \\ University of Rochester \\ Rochester, NY 14618 \\ U.S.A. \\ Email: wth2@mail.rochester.edu. *
}

May, 2007; this version July 15, 2010

${ }^{*}$ I thank a referee, Tarık Kara, Yoichi Kasajima, and John Weymark for their comments, and the NSF for its support under grant No. SES 0214691. This article was prepared for presentation at a conference honoring Serge-Christophe Kolm held at the University of Caen in May 2007. BorrowLindahl.tex 


\begin{abstract}
In the context of a simple model of public good provision, we study the requirement on an allocation rule that it be immune to manipulation by augmenting one's endowment through borrowing from the outside world. We call it open-economy borrowing-proofness (Thomson, 2009). We ask whether the Lindahl rule satisfies the property. The answer is yes on both the domain of quasi-linear economies and on the domain of homothetic economies. However, on the classical domain (when preferences are only required to be continuous, monotone, and convex), the answer is negative. We compare the manipulability of the rule through borrowing and its manipulability through withholding. We also asks whether the rule is immune to manipulation by borrowing from a fellow trader, closed-economy borrowing-proofness. We obtain a parallel set of answers. The negative results hold no matter how small the amount borrowed is constrained to be.
\end{abstract}

Key-words: Public good; Lindahl rule; Kolm triangle; borrowing-proofness; withholding-proofness. 


\section{Introduction}

Allocation rules can be manipulated in a variety of ways. Agents may misrepresent their preferences, a possibility that has been extensively studied. They can also take advantage of the control they have over resources, and different kinds of stratagems of this type have been identified. For instance, in a classical exchange economy in which resources are allocated by means of the Walrasian rule, an agent may benefit from withholding some of his endowment. He may even benefit from destroying some of it. Also, he may benefit from transferring some of his endowment to someone else, (of course without this second agent being hurt, thereby having no reason not to accept the transfer). For a discussion of these phenomena, see Gale (1974), Aumann and Peleg (1974), Postlewaite (1979), and Thomson (1987a,b). Additional manipulation opportunities are discussed in Thomson (2007).

We consider here the following form of manipulation. Suppose that prior to the operation of the chosen rule, an agent borrows resources so as to augment his endowment. The rule is then applied; he receives the consumption bundle the rule assigns to him, and he returns what he had borrowed. In the end, he may be better off than if he had not borrowed. Our objective is to study the requirement on a rule that it not be subject to this kind of manipulation. We call it "borrowing-proofness". The requirement is formulated and studied in the context of classical economies by Thomson (2009).

We have in mind two possible scenarios. First, an agent may have access to resources outside of the group of agents with whom he normally trades. There is a growing literature concerning situations of this kind, when an agent may exploit the particular place he holds in the social network. Here, we imagine that an agent who is part of some community is also involved with another community where he can borrow resources. This other community is not explicitly modeled. We then have an "open-economy" model, and we refer to the property of immunity to manipulation through borrowing from the outside world as "open-economy borrowing-proofness". Alternatively, the agent could simply exaggerate the resources he owns, without the social planner being able to verify these claims of ownership. This virtual augmentation is mathematically equivalent to actual borrowing from the outside, but for simplicity we will mainly refer to one story, the borrowing story.

The second scenario pertains to situations in which an agent borrows from

one of his fellow traders. He should of course provide the lender the incentive to do so: after the borrower has returned what he borrowed, the lender should 
be at least as well off as he would have been if he had not lent. We now have a "closed-economy" model and we refer to the property of immunity to borrowing from within as "closed-economy borrowing-proofness".

The resource allocation problem we consider is standard: there are two goods, one private good and one public good; the private good can be either consumed as such, or it can be used as input in the production of the public good. At what level should the public good be produced, and how much should each agent contribute to its production? These are the questions addressed in the literature on public good provision.

We investigate the manipulability through borrowing of a rule that is central to this literature, the "Lindahl rule" (Lindahl, 1919). Characterizing the equilibria of manipulation games associated with this rule when agents can either withhold part of their endowments or exaggerate them is the object of Thomson (1979) and Sertel and Sanver (1999). We study the borrowing-proofness of the Lindahl rule on three standard preference domains, when only the "classical" assumptions of continuity, monotonicity, and convexity of preferences are made, and when in addition, preferences are either quasi-linear or homothetic. (We add assumptions that guarantee its single-valuedness.) We show that on both the quasi-linear and the homothetic domains, the Lindahl rule is borrowing-proof under either scenario (Propositions 1 and 2 and Propositions 3 and 4). In fact, as long as the public good is a normal good, this conclusion holds. However, on the classical domain, it does not (Examples 1 and 4).

It is interesting to relate open-economy borrowing-proofness to the requirement of immunity to manipulation through withholding of some of one's endowment, called "withholding-proofness" (Postlewaite, 1979, introduces this property for private good economies). It is known that the Lindahl rule is not withholding-proof on the classical domain (Thomson, 1979, 1987b). We show that this negative result also holds on either the quasi-linear domain or the homothetic domain (Examples 2 and 3). Altogether then, manipulation through open-economy borrowing should be less of a concern than manipulation through withholding.

Another reason why it should be less of a concern is of course that borrowing requires an agent to have access to external resources under the first scenario, and to convince one of his fellow traders to go along with his scheme under the second scenario. By contrast, withholding can be carried out entirely on one's own. When manipulation is interpreted as exaggeration, asking agents to provide proof of ownership is a practical way to deal with the 
behavior.

Yet, for our negative results, we place a bound on the amount borrowed, and in fact, we prove stronger claims: no matter how small that bound is, borrowing-proofness fails.

Why should we worry about agents manipulating through borrowing? For the same reason we should worry about them manipulating by misrepresenting their preferences, or by transferring their endowments among one another, or by withholding or destroying part of their endowments. An allocation rule is chosen because of the properties it enjoys. If an agent does not behave as specified by the rule, the allocations the rule is supposed to reach are less likely to be reached. In most cases, the first consequence of manipulation, be it misrepresentation of preferences or endowment manipulation in any of its forms, is that a rule designed to produce efficient allocations will be prevented from doing so. Also, a rule is usually specified so as to satisfy some distributional or participation requirements. These too may not be satisfied if agents manipulate.

Ours is not an intertemporal model of a monetary economy. Borrowing financial resources is of course a very convenient means of achieving intertemporal efficiency and there is no reason to object to it in that context. ${ }^{1}$

To establish most of our results, we use the so-called Kolm triangle, the very ingenious geometric representation of public good economies with two agents, two goods, one private good and one public good, and a linear technology, developed by Kolm (1971). Throughout this exposition we adopt the didactic style that this device makes possible. ${ }^{2}$

The article is organized as follows. In Section 2, we introduce the model. In Section 3, we define and discuss the open-economy version of borrowingproofness. In Section 4, we study the manipulability of the Lindahl rule through withholding and provide some insight into the reasons why it is more vulnerable to this sort of behavior than to manipulation through openeconomy borrowing. In Section 5, we consider the closed-economy version of borrowing-proofness. In Section 6, we offer concluding comments and list

\footnotetext{
${ }^{1}$ Unfortunately, in describing the phenomenon in which we are interested, we could not find a term that would not bring to mind allocation over time.

${ }^{2}$ Other geometric representations have been proposed. The Dolbear (1967) triangle allows one to keep standard rectangular axes for one of the agents but not for both. Thus, it breaks the symmetry between them. The Kolm triangle has the advantage of treating both agents symmetrically. The representation used by Sertel (1994) uses standard rectangular axes for both agents, but a feasible allocation is mapped into two points in $\mathbb{R}_{+}^{2}$.
} 
open questions.

\section{Notation and definitions}

There are two goods, one private good and one public good, and $n \in \mathbb{N}$ agents. Let $N \equiv\{1, \ldots, n\}$ be the set of agents. Each agent $i \in N$ is equipped with a preference relation defined on $\mathbb{R}_{+}^{2}$, denoted by $R_{i}$. Let $P_{i}$ denote the strict preference relation associated with $R_{i}$ and $I_{i}$ the corresponding indifference relation. Let $R \equiv\left(R_{1}, \ldots, R_{n}\right)$ denote the profile of preferences. Each agent $i \in N$ is endowed with an amount $\omega_{i x} \geq 0$ of the private good. Let $\omega_{x} \equiv\left(\omega_{1 x}, \ldots, \omega_{n x}\right) \in \mathbb{R}_{+}^{N}$ denote the profile of these endowments. ${ }^{3}$ The private good can be consumed as such or it can be used in the production of the public good. Initially, there is no public good, so we set $\omega_{y} \equiv 0$. Let $\omega \equiv\left(\omega_{x}, \omega_{y}\right) \in \mathbb{R}_{+}^{N} \times \mathbb{R}_{+}$. The production set is a subset of $\mathbb{R}^{2}$ denoted $Y$, with inputs measured negatively and outputs measured positively. An economy with agent set $\boldsymbol{N}$ is a list $(R, \omega, Y)$ in some domain $\mathcal{E}^{N}$ defined by imposing restrictions on preferences (such as continuity and convexity) and on the production set (such as convexity). A (feasible) allocation for $(\boldsymbol{R}, \boldsymbol{\omega}, \boldsymbol{Y}) \in \mathcal{E}^{\boldsymbol{N}}$ is a list $z \equiv(x, y) \in \mathbb{R}_{+}^{N} \times \mathbb{R}_{+}$ such that $\left(-\sum\left(\omega_{i x}-x_{i}\right), y\right) \in Y$. Agent $\boldsymbol{i}$ 's consumption at $\boldsymbol{z}$ is the pair $z_{i} \equiv\left(x_{i}, y\right)$. Let $\boldsymbol{Z}(\boldsymbol{\omega}, \boldsymbol{Y})$ denote the set of feasible allocations of $(R, \omega, Y)$. We work with a linear technology: there is $\alpha \in \mathbb{R}_{+}$such that the production of each unit of the public good requires $\alpha$ units of input. We choose units of measurement of the goods so that $\alpha=1$.

Our results pertain to the classical domain, when preferences are continuous, convex, and monotonic (an increase in the consumption of any of the goods makes an agent better off), and two important subdomains, defined as follows. The preference relation $R_{i}$ is quasi-linear if indifference between bundles is preserved under translations of these bundles parallel to the private good axis by any non-negative amount: for each pair $\left\{z_{i}, z_{i}^{\prime}\right\} \in \mathbb{R}_{+}^{2}$ and each $t \in \mathbb{R}_{+}$, if $z_{i} I_{i} z_{i}^{\prime}$, then $\left(z_{i}+(t, 0)\right) I_{i}\left(z_{i}^{\prime}+(t, 0)\right) .{ }^{4}$ The preference

\footnotetext{
${ }^{3}$ The notation $\mathbb{R}^{N}$ and $\mathbb{R}_{+}^{N}$ designates the cross-product of $|N|$ copies of $\mathbb{R}$ and $\mathbb{R}_{+}$ respectively indexed by the elements of $N$.

${ }^{4} \mathrm{~A}$ common alternative specification of the quasi-linearity assumption is obtained by dropping the requirement that the consumption of the private good be non-negative, and in fact, letting it be unbounded below. Then, the Pareto set is invariant under arbitrary (in sign and magnitude) transfers of the private good. This makes working with this model
} 
relation $R_{i}$ is homothetic if indifference between bundles is preserved under homothetic transformations of these bundles: for each pair $\left\{z_{i}, z_{i}^{\prime}\right\} \in \mathbb{R}_{+}^{2}$ and each $\lambda \in \mathbb{R}_{+}$, if $z_{i} I_{i} z_{i}^{\prime}$, then $\lambda z_{i} I_{i} \lambda z_{i}^{\prime}$. Let $\boldsymbol{\mathcal { R }}_{\boldsymbol{c l}}$ denote the domain of classical preferences. Let $\boldsymbol{\mathcal { R }}_{\boldsymbol{q l}}$ and $\boldsymbol{\mathcal { R }}_{\text {hom }}$ denote the domain of preferences, that, in addition to satisfying the classical assumptions, are quasi-linear and homothetic respectively. Let $\mathcal{E}_{\boldsymbol{c l}}^{N}, \mathcal{E}_{\boldsymbol{q l}}^{\boldsymbol{N}}$, and $\mathcal{E}_{\text {hom }}^{\boldsymbol{N}}$ be the corresponding domains of economies. Let $\mathcal{E}^{N}$ be a generic domain of economies.

Given a domain $\mathcal{E}^{N}$, a solution on $\mathcal{E}^{N}$ is a mapping $\varphi$ associating with each $(R, \omega, Y) \in \mathcal{E}^{N}$ a non-empty subset of $Z(\omega, Y)$, denoted $\varphi(R, \omega, Y)$. We use the term rule when the mapping is single-valued. A solution $\varphi$ is essentially single-valued if for each $(R, \omega, Y) \in \mathcal{E}^{N}$, each pair $\left\{z, z^{\prime}\right\} \subseteq$ $\varphi(R, \omega, Y)$, and each $i \in N, z_{i} I_{i} z_{i}^{\prime} .5$ The Pareto solution, $\boldsymbol{P}$, associates with each economy its set of allocations such that there is no other allocation that each agent finds at least as desirable, and at least one agent prefers: $z \in P(R, \omega, Y)$ if $z \in Z(\omega, Y)$ and there is no $z^{\prime} \in Z(\omega, Y)$ such that for each $i \in N, z_{i}^{\prime} R_{i} z_{i}$, and for at least one $i \in N, z_{i}^{\prime} P_{i} z_{i}$.

Given $a^{1}, a^{2} \in \mathbb{R}_{+}^{2}$, we designate by $\operatorname{seg}\left[\boldsymbol{a}^{\mathbf{1}}, \boldsymbol{a}^{\mathbf{2}}\right]$ the segment connecting these two points. Given $a^{1}, \ldots, a^{k} \in \mathbb{R}_{+}^{2}$, we designate by bro.seg $\left[\boldsymbol{a}^{\mathbf{1}}, \ldots, \boldsymbol{a}^{\boldsymbol{k}}\right]$ the broken segment $\operatorname{seg}\left[a^{1}, a^{2}\right] \cup \cdots \cup \operatorname{seg}\left[a^{k-1}, a^{k}\right]$.

\section{Open-economy borrowing-proofness}

Next is a formal statement of our requirement of immunity to manipulation through borrowing from the outside. Agent $i$ is the borrower and he augments his endowment of the private good from $\omega_{i x}$ to $\omega_{i x}^{\prime}$ by borrowing. (Obviously, one cannot borrow a public good.) Given $\omega_{x} \in \mathbb{R}_{+}^{N}$ and $i \in N$, we denote by $\omega_{-i x}$ the vector obtained from $\omega_{x}$ by dropping its $i$-th coordinate. Let $\varphi$ be a solution.

Open-economy borrowing-proofness: For each $e \equiv(R, \omega, Y) \in \mathcal{E}^{N}$, each $i \in N$, and each $\omega^{\prime} \in \mathbb{R}_{+}^{N} \times \mathbb{R}_{+}$, if $\omega_{i x}^{\prime}>\omega_{i x}, \omega_{-i x}^{\prime}=\omega_{-i x}$, and $\omega_{y}^{\prime}=0$, then it is not the case that for some $z \in \varphi(R, \omega, Y)$ and for some $z^{\prime} \in \varphi\left(R, \omega^{\prime}, Y\right),\left[z_{i}^{\prime}-\left(\omega_{i x}^{\prime}-\omega_{i x}, 0\right)\right] P_{i} z_{i}$.

particularly easy.

${ }^{5}$ We could also speak of a rule being single-valued up to Pareto-indifference. 
For an agent to want to borrow, he should first be able to return what he borrowed. Thus, the property is written in the negative. We should worry about him borrowing only if he is able to return what he borrowed and after doing so, ends up better off.

We could have stated the definition for single-valued solutions. In the strategic analysis of allocation correspondences, to each strategy is associated a set of outcomes, and the question comes up how a player bases his choice of a strategy on comparisons of sets of outcomes. This question does not arise for a single-valued solution, that is, for what we called a "rule". We wrote our definition with existential quantifiers on the allocations chosen before and after the manipulation. This means that no matter what choice would be made if he did not borrow, an agent would evaluate optimistically the choice that would be made if he did borrow. However, our results are proved in situations in which the solution that interests us here is single-valued. ${ }^{6}$

An alternative definition is obtained by imagining that the borrower obtains resources from one of his fellow traders, instead of from an unspecified outside world. In general, the bundle the rule assigns to the lender will change when the rule is operated from the reshuffled endowments, and he may find his new bundle worse than the bundle he would have received if he had not lent. For him to accept lending, it should be the case that after getting back the resources he lent, he does end up at least as well off as if he had not lent. The possibility of improving one's welfare by borrowing "from within" is discussed in Section 5.

We prove our negative results and illustrate our positive ones concerning open-economy borrowing-proofness by means of two-agent examples. Then, we can use the geometric device known as the Kolm triangle (Kolm, 1970). We assume familiarity with this device (for a didactic exposition, see Thomson, 1999). The essential features are as follows. Let $N \equiv\{1,2\}$ and $e \equiv(R, \omega, Y) \in \mathcal{E}^{N}$ be an economy with a linear (normalized, that is, each unit of output requiring one unit of input) technology. An allocation $z \equiv\left(x_{1}, x_{2}, y\right) \in Z(\omega, Y)$ is represented as a point in an equilateral triangle of height $\omega_{1 x}+\omega_{2 x}$. The lower left and lower right corners, labeled $0_{1}$

\footnotetext{
${ }^{6}$ One could allow for essential single-valuedness, but Pareto-indifference of the allocations chosen for the reported profile of endowments would not necessarily imply Paretoindifference after the amounts borrowed have been returned. Thus, the problem of specifying how an agent compares sets of allocations would still have to be faced. The issue is discussed by Thomson (1979). A concept of immunity to manipulation for correspondences is studied by Ching and Zhou (2002), but other formulations have been proposed.
} 
and $\mathrm{O}_{2}$, are origins. The two agents' consumptions are measured from these points. Their consumptions $x_{1}$ and $x_{2}$ of the private good are measured by the distances from $z$ to the left and right sides of the triangle (and not by the horizontal distances from $z$ to these sides); their common consumption of the public good is measured by the vertical distance from $z$ to the base of the triangle.

On either the quasi-linear domain or the homothetic domain, the Pareto set has a simple structure, even more so if in fact preferences are strictly convex, as depicted in our figures.

The key implication of quasi-linearity for convex preferences, in either the standard rectangular axes or in the slanted axes of the Kolm triangle representation, is that for each agent $i \in N$ and each pair of bundles $z_{i} \equiv$ $\left(x_{i}, y\right)$ and $z_{i}^{\prime} \equiv\left(x_{i}^{\prime}, y^{\prime}\right)$, the line(s) of support to his upper contour set at $z_{i}$ is (are) at least as steep as the line(s) of support to his upper contour set at $z_{i}^{\prime}$ if and only if $y \geq y^{\prime}$. If preferences are strictly convex and $z$ is an efficient allocation, no other allocation is Pareto-indifferent to it. Then, in the Kolm triangle, under quasi-linearity, the Pareto set has three parts, which to save space, we do not show on a separate figure, but on Figure 3, used later to prove one of our results. Consider the triangle with origins $0_{1}$ and $0_{2}$ in that figure. The true endowment profile corresponds to the point marked $\omega$. For each $i \in N, z^{i}$ designates the most preferred allocation for agent $i$ in that triangle. The three parts then are (i) a horizontal segment from the left side of the triangle to the right side, $\operatorname{seg}\left[a^{1}, a^{2}\right]$, at each point of which indifference curves admit a common line of support; (ii) a segment lying in the left side of the triangle, $\operatorname{seg}\left[z^{2}, a^{1}\right]$, and (iii) a segment lying in its right side, $\operatorname{seg}\left[z^{1}, a^{2}\right]$.

Given $z_{i} \in \mathbb{R}_{+}^{2} \backslash\left\{0_{i}\right\}$, we designate by $\boldsymbol{\rho}\left(\mathbf{0}_{\boldsymbol{i}}, \boldsymbol{z}_{\boldsymbol{i}}\right)$ the ray emanating from agent $i$ 's origin $0_{i}$ and passing through $z_{i}$. The key implication of homotheticity for convex preferences is that for each agent $i \in N$ and each pair of bundles $z_{i} \equiv\left(x_{i}, y\right)$ and $z_{i}^{\prime} \equiv\left(x_{i}^{\prime}, y^{\prime}\right)$, the line(s) of support to his upper contour set at $z_{i}$ is (are) at least as steep as the line(s) of support to his upper contour set at $z_{i}^{\prime}$ if and only if $\rho\left(0_{i}, z_{i}\right)$ is at least as steep as $\rho\left(0_{i}, z_{i}^{\prime}\right)$. In the Kolm triangle, under strict convexity and homotheticity, the Pareto set is a curve connecting the slanted sides of the triangle that satisfies the following property. If $z$ is an efficient allocation, the rest of the Pareto set lies entirely in the bow-tie shaped area between the rays emanating from the two origins 
and passing through $z, \rho\left(0_{1}, z_{1}\right)$ and $\rho\left(0_{2}, z_{2}\right){ }^{7}$

The solution defined by Lindahl (1919) can be considered as a counterpart for public good economies of the Walrasian rule, as it calls for each agent to maximize his preferences on a budget line. The main difference is that prices for public goods (in terms of the private good) are individualized. The producer faces a price for the public good that is the sum of the prices faced by the consumers. Profits made in production are distributed according to a vector $\theta \in \Delta^{N}$ of profit shares. The Lindahl solution, $\boldsymbol{L}$, associates with each economy its set of allocations that can be supported by prices: $z \equiv(x, y) \in L(R, \omega, Y)$ (here, the technology $Y$ does not have to be linear) if $z \in Z(\omega, Y)$ and there is $p \equiv\left(p_{i}\right)_{i \in N} \in \mathbb{R}_{+}^{N}$ such that for each $i \in N, x_{i}+p_{i} y \leq$ $\omega_{i x}$, and for each $z_{i}^{\prime} \equiv\left(x_{i}^{\prime}, y^{\prime}\right) \in \mathbb{R}_{+}^{2}$ such that $x_{i}^{\prime}+p_{i} y^{\prime} \leq \omega_{i x}+\theta_{i}\left(-1, \sum p_{i}\right) z^{*}$, where $z^{*} \in Y$ is a production plan maximizing $\left(-1, \sum p_{i}\right) z$ for $z \in Y$, we have $z_{i} \quad R_{i} z_{i}^{\prime}$. The prices $p$ are called "equilibrium prices". An agent's "offer curve" is defined as in Walrasian analysis, by tracing out the agent's maximizing bundle as a function of the prices he faces. Let agent $i$ 's offer curve from endowment $\omega_{i}$ be denoted $\boldsymbol{o c}\left(\boldsymbol{R}_{\boldsymbol{i}}, \boldsymbol{\omega}_{\boldsymbol{i}}\right)$ (preferences being fixed, in the figures, we use the more compact notation $\left.\boldsymbol{o c}\left(\boldsymbol{\omega}_{\boldsymbol{i}}\right)\right)$. In the Kolm triangle, the Lindahl allocations are obtained in the same way that Walrasian allocations are in the Edgeworth box, by intersecting offer curves.

We specify the counterexamples used for our negative results geometrically, introducing them in such a way as to make it as intuitive as possible how we arrived at them. We do not give explicit analytical expressions for representations of preferences. Such expressions would often be quite complicated without shedding any additional light on the nature of the results.

Figure 1 shows how to accommodate borrowing. We always consider borrowing by agent 1 , calling $\beta>0$ the amount he borrows. We need to enlarge the triangle by this amount. We keep his origin fixed at $0_{1}$, and translate agent 2's origin to the right by the vector $\left(\frac{2}{\sqrt{3}} \beta, 0\right)$, denoting his new origin $0_{2}^{\prime}$. We also translate the endowment profile and any data that pertains to agent 2 in the original triangle (indifference curves, consumptions)

\footnotetext{
${ }^{7}$ The proof is simple. Let $z \in P(e)$. In the Kolm triangle, the lines of support to the two agents' indifference curves at $z$ coincide. Let $z^{\prime}$ be another feasible allocation. If $z^{\prime}$ is above $\rho\left(0_{1}, z_{1}\right)$, agent 1 's line(s) of support at $z_{1}^{\prime}$ is (are) steeper than its line(s) of support at $z_{1}$. If $z^{\prime}$ is also above $\rho\left(0_{2}, z_{2}\right)$, agent 2's line(s) of support at $z_{2}^{\prime}$ is (are) flatter than its line(s) of support at $z_{2}$. Thus, the lines of support at $z_{1}^{\prime}$ and $z_{2}^{\prime}$ cross. The same argument can be made about any point that is below both $\rho\left(0_{1}, z_{1}\right)$ and $\rho\left(0_{2}, z_{2}\right)$. The remaining points are between $\rho\left(0_{1}, z_{1}\right)$ and $\rho\left(0_{2}, z_{2}\right)$, as claimed.
} 


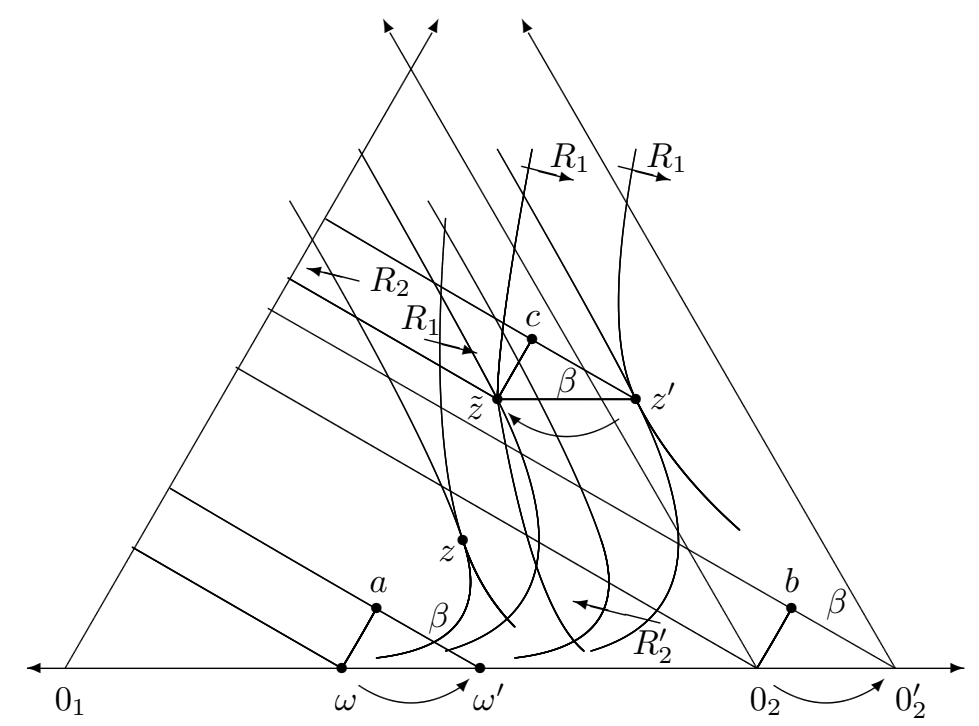

Figure 1: Enlarging a Kolm triangle so as to accommodate borrowing.

by this vector, since all of these objects have to be re-measured from $0_{2}^{\prime}$. Let $\operatorname{tr}\left(\boldsymbol{\omega}, \boldsymbol{\omega}^{\prime}\right)$ be this horizontal translation. In the figure, the segments $\operatorname{seg}\left[a, \omega^{\prime}\right]$, $\operatorname{seg}\left[b, 0_{2}^{\prime}\right]$, and $\operatorname{seg}\left[c, z^{\prime}\right]$ all have length $\beta$. Suppose a rule selects $z$ for $(R, \omega, Y)$ and $z^{\prime}$ for $\left(R, \omega^{\prime}, Y\right)$. Agent 1's final consumption, once he has returned what he borrowed, is the pair consisting of the first and third components of the allocation $\tilde{z}$ obtained by translating $z^{\prime}$ by the vector $\left(-\frac{2}{\sqrt{3}} \beta, 0\right)$ (this is the translation $\left.-\operatorname{tr}\left(\omega, \omega^{\prime}\right)\right)$. We now compare $z_{1}$ to $\tilde{z}_{1} \equiv z_{1}^{\prime}-(\beta, 0)$. For the rule to be open-economy borrowing-proof, $z_{1}$ should always be at least as desirable as $\tilde{z}_{1}$ according to $R_{1}$. In Figure 1 , that is not the case, so the property is violated.

The figure shows that even if a rule is designed to select efficient allocations (note that indifference curves are tangent at $z$ in the original triangle and also at $z^{\prime}$ in the enlarged triangle), the allocation that results after the borrower has returned the resources he borrowed may not be efficient. Indeed, the indifference curves through $\tilde{z}$ are typically not tangent there. This is of course one of the reasons why we should be concerned about borrowing.

We show that on both the quasi-linear domain and on the homothetic domain, the Lindahl rule is open-economy borrowing-proof.

On the quasi-linear domain, we may think that if an allocation is efficient, so is any other allocation obtained by horizontal translation to the left or to the right, and that translation invariance of any rule would imply its open-economy borrowing-proofness. However, one should be careful about boundaries, and even on its subdomain on which it selects interior allocations, a rule need not be translation invariant. The Lindahl rule does satisfy this 


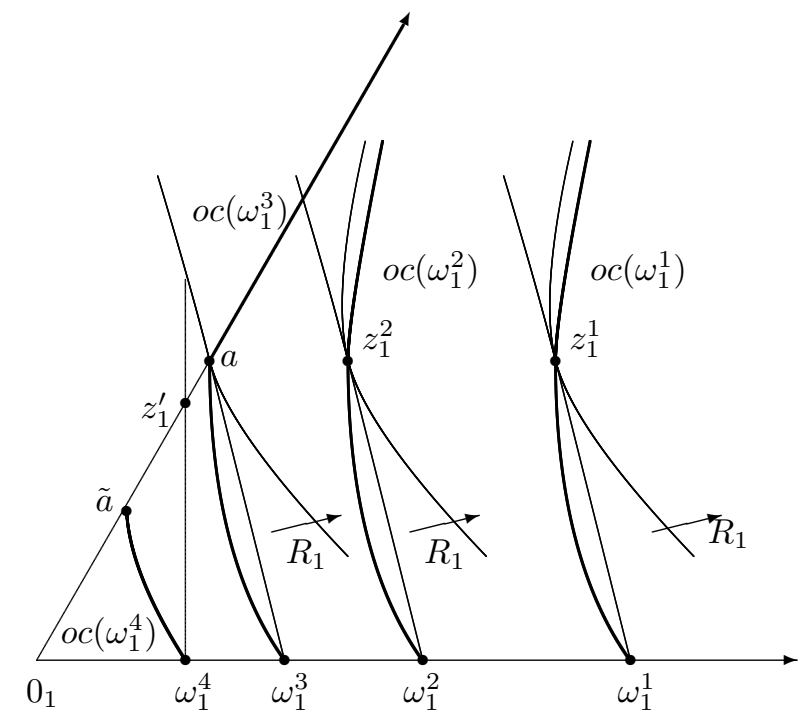

Figure 2: Offer curves for quasi-linear preferences.

property whenever it selects interior allocations, but other appealing rules exist that do not.

Figure 2 shows what happens to an agent's offer curve as his endowment decreases. As we will soon embed agent 1's map into a Kolm triangle, let us work within slanted axes. For the endowment $\omega_{1}^{1}$, agent 1 's offer curve never reaches the slanted axis $^{8}$ and that is also the case for any endowment to the right of $\omega_{1}^{1}$ and for a range of endowments to the left of $\omega_{1}^{1}$, such as $\omega_{1}^{2}$. The bundles maximizing his preferences on two parallel price lines, if both bundles are interior, are related by a horizontal translation. Examples are the points $z_{1}^{1}$ and $z_{1}^{2}$, which maximize his preferences on two parallel budget lines emanating from $\omega_{1}^{1}$ and $\omega_{1}^{2}$. His offer curve for the endowment $\omega_{1}^{3}$ is obtained by translating his offer curve for $\omega_{1}^{1}$ and truncating it by the slanted axis: in the figure, it consists of the arc from $\omega_{1}^{3}$ to $a$ and the part of that axis that is above $a$. For the even smaller endowment $\omega_{1}^{4}$, the truncation is more significant and the offer curve consists of the arc from $\omega_{1}^{4}$ to $\tilde{a}$ and the part of the left slanted axis that is above $\tilde{a}$. The point $z_{1}^{\prime}$ is a maximizer of the agent's preferences on a price line emanating from $\omega_{1}^{4}$.

On the quasi-linear domain with strictly convex and smooth preferences, the Lindahl correspondence is single-valued. This conclusion holds because each agent's demand for the public good is a monotonic function of the price of that good (relative to that of the private good) that he faces.

\footnotetext{
${ }^{8}$ For some preference relations, all offer curves reach the slanted axis.
} 


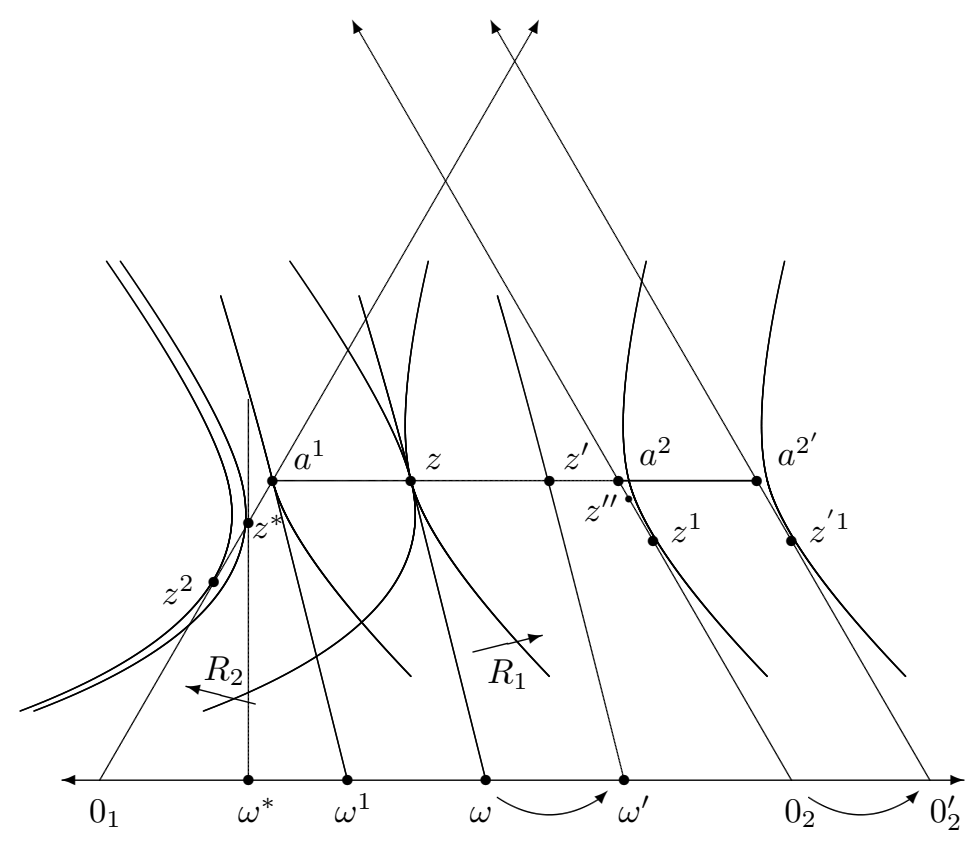

Figure 3: On the domain of strictly convex, smooth, and quasi-linear preferences, the Lindahl rule is open-economy borrowing-proof (Proposition 1).

Proposition 1 On the domain of strictly convex, smooth, and quasi-linear preferences, the Lindahl rule is open-economy borrowing-proof.

Proof: The proof is illustrated in Figure 3 for a strictly convex and smooth economy $(R, \omega, Y) \in \mathcal{E}_{q l}^{N}$, where $N \equiv\{1,2\}$. The proof for more than two agents is essentially the same and we omit it. Let $z \equiv\left(x_{1}, x_{2}, y\right) \equiv$ $L(R, \omega, Y)$. Agent 1 borrows the amount $\beta$ of the private good. Let $\omega^{\prime}$ be the new endowment profile in the enlarged Kolm triangle and $z^{\prime} \equiv L\left(R, \omega^{\prime}, Y\right)$. If $x_{1}>0$ (as illustrated in the figure), then $z_{1}^{\prime}=z_{1}+(\beta, 0)$. Indeed, by the translation invariance of indifference curves, the price line that makes $z$ a Lindahl allocation in the original economy, when translated by the amount borrowed, is a price line that makes the translate of $z$ by $\operatorname{tr}\left(\omega, \omega^{\prime}\right)$ a Lindahl allocation in the augmented economy: the allocation chosen by the Lindahl rule moves in a co-variant way with agent 1's borrowing. After returning the amount borrowed, he ends up with the same bundle as he would have received had he not borrowed. (If agent 2's consumption of the private good is zero initially, such as at $z^{\prime \prime}$ in the figure, the argument is the same as for interior Lindahl allocations.)

For an endowment profile sufficiently close to $0_{1}$, such as $\omega^{*}$ in the figure, the resulting Lindahl allocation $z^{*} \equiv L\left(R, \omega^{*}, Y\right)$ is a boundary allocation $\left(x_{1}^{*}=0\right)$. If agent 1 borrows an amount that brings the endowment allocation to a point such as $\omega^{1}$, the new Lindahl allocation is $a^{1}$, a boundary allocation too $\left(x_{1}=0\right)$. Agent 1 is obviously unable to return what he borrowed. The 


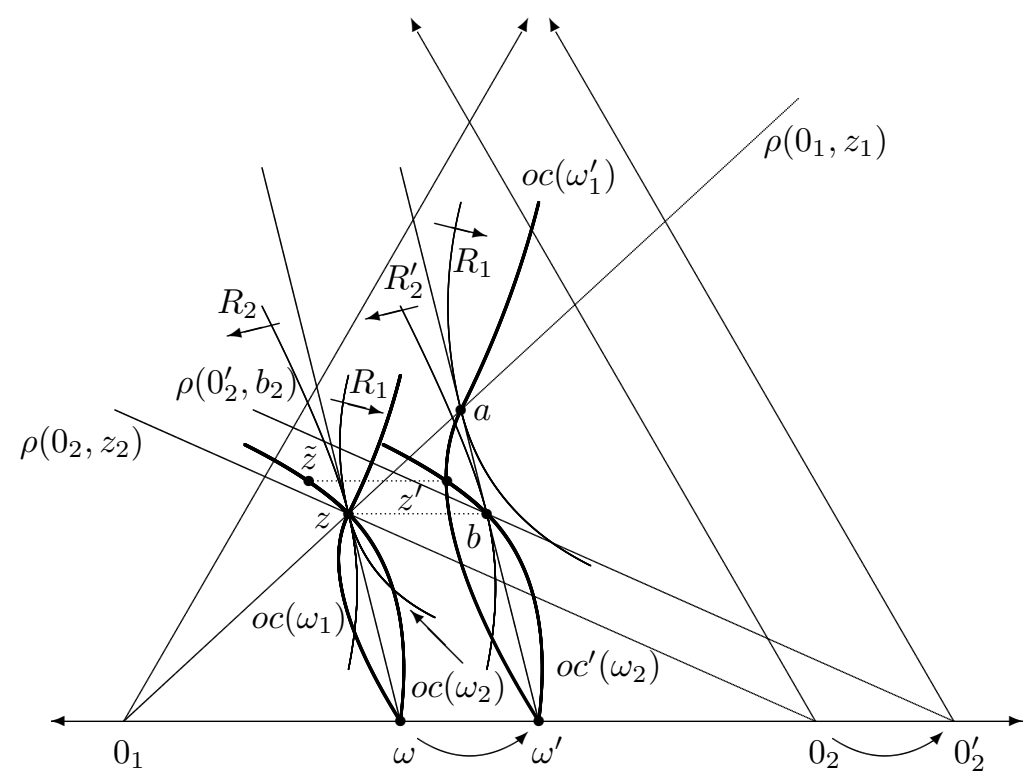

Figure 4: On the domain of strictly convex and homothetic preferences, the Lindahl rule is open-economy borrowing-proof (Proposition 2).

same observation holds for any borrowed amount that brings the endowment allocation to a point of $\operatorname{seg}\left[\omega^{*}, \omega^{1}\right]$, the resulting Lindahl allocation being a point of $\operatorname{seg}\left[z^{*}, a^{1}\right]$. If he borrows enough for the resulting Lindahl allocation to be interior - for instance, if the new endowment allocation is $\omega$, the Lindahl allocation is $z$-but he ends up paying more per unit of the public good than he did initially, and he receives less of the private good than he borrowed. Thus, his new assignment is still insufficient for him to return what he borrowed.

On the homothetic domain with strictly convex preferences, the Lindahl rule is single-valued. Just like for the quasi-linear case, this is because each agent's demand for the public good is a strictly decreasing function of the price of that good (relative to that of the private good) that he faces.

Proposition 2 On the domain of strictly convex and homothetic preferences, the Lindahl rule is open-economy borrowing-proof.

Proof: The proof is illustrated in Figure 4 for a strictly convex economy $(R, \omega, Y) \in \mathcal{E}_{\text {hom }}^{N}$, where $N \equiv\{1,2\}$. The proof for more than two agents is essentially the same. Let $z \equiv L(R, \omega, Y)$. Agent 1 borrows the amount $\beta$ of the private good. Let $\omega^{\prime}$ be the new endowment profile in the enlarged Kolm triangle and $z^{\prime} \equiv L\left(R, \omega^{\prime}, Y\right)$. Agent 1's offer curve from $\omega_{1}^{\prime}$, oc $\left(\omega_{1}^{\prime}\right)$, goes through the point $a$ obtained by subjecting $z$ to the same homothetic expansion centered at $0_{1}$ that takes $\omega$ to $\omega^{\prime}$. Also, agent 2's offer curve 


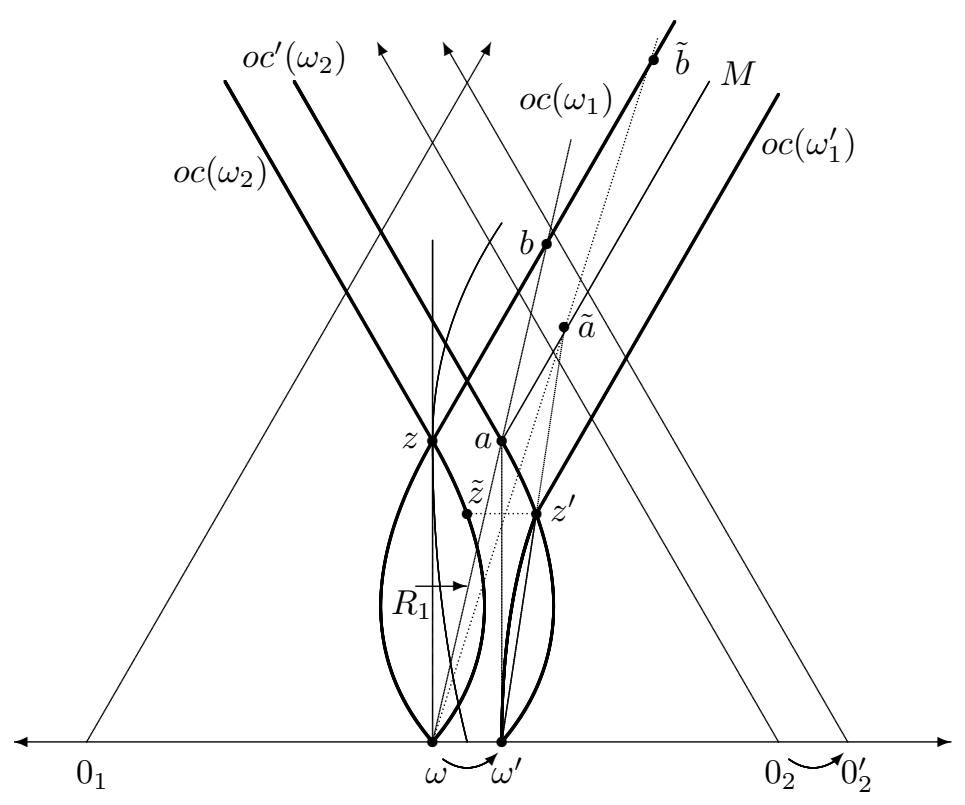

Figure 5: On the classical domain, the Lindahl rule is not open-economy borrowing-proof (Example 1).

measured from $0_{2}^{\prime}$ is obtained by subjecting $o c\left(\omega_{2}\right)$ to $\operatorname{tr}\left(\omega, \omega^{\prime}\right)$. The point $b$ is the image of $z$ under that translation. It follows from these facts that $o c\left(\omega_{1}^{\prime}\right)$ and $o c^{\prime}\left(\omega_{2}\right)$ cross at a point $z^{\prime}$ to the left of the line obtained by subjecting the initial equilibrium price line to $\operatorname{tr}\left(\omega, \omega^{\prime}\right)$. Agent 1's final consumption is obtained by subjecting $z_{1}^{\prime}$ to the inverse translation, $-\operatorname{tr}\left(\omega, \omega^{\prime}\right)$, and it is therefore to the left of the initial equilibrium price line. Agent 1 does not benefit from borrowing.

It is not difficult to see that as long as the public good is a normal good, the reasoning just made applies. In their analysis of the endowment misrepresentation game associated with the Lindahl rule, Sertel and Sanver (1999) note that if the public good is a normal good, then at equilibrium, agents do not exaggerate their endowments. This result implies the conclusions that we have reached by means of our graphical analysis.

Finally, we consider the classical domain and show by means of an economy with strictly convex preferences (Example 1) that, now, open-economy borrowing-proofness fails.

Example 1 On the strictly convex and classical domain, the Lindahl rule is not open-economy borrowing-proof. The proof is by means of a strictly convex economy $(R, \omega, Y) \in \mathcal{E}_{c l}^{N}$, where $N \equiv\{1,2\}$, illustrated in Figures 5 and 6. Agent 1's intended offer curve from $\omega_{1}, o c\left(\omega_{1}\right)$, consists of a backwardbending arc from $\omega_{1}$ to a point $z$ on the vertical line through $\omega$, followed by a half-line parallel to the left side of the triangle. Agent 2's preferences are 


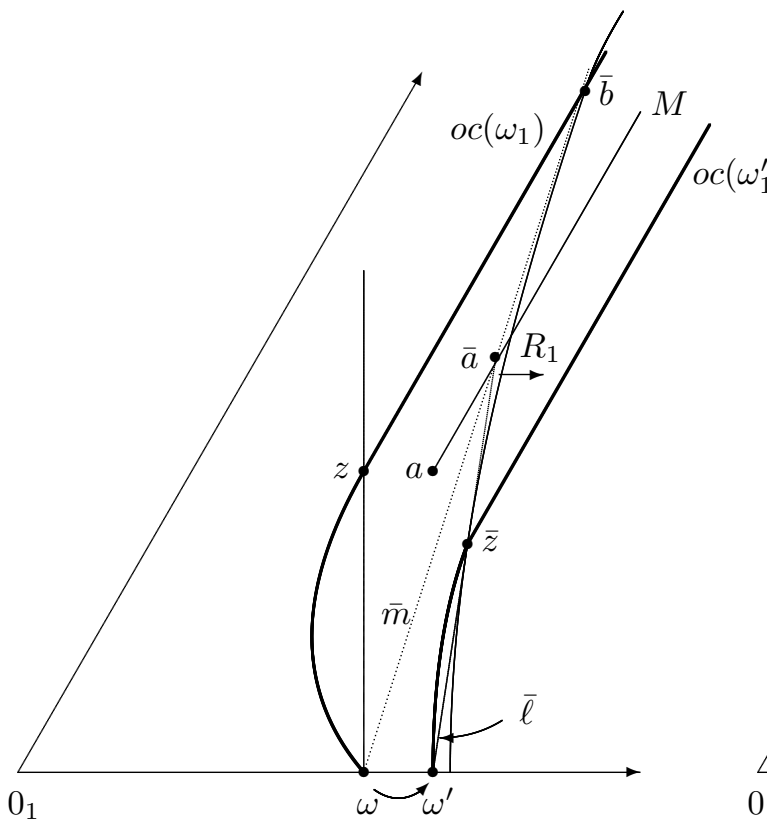

(a)

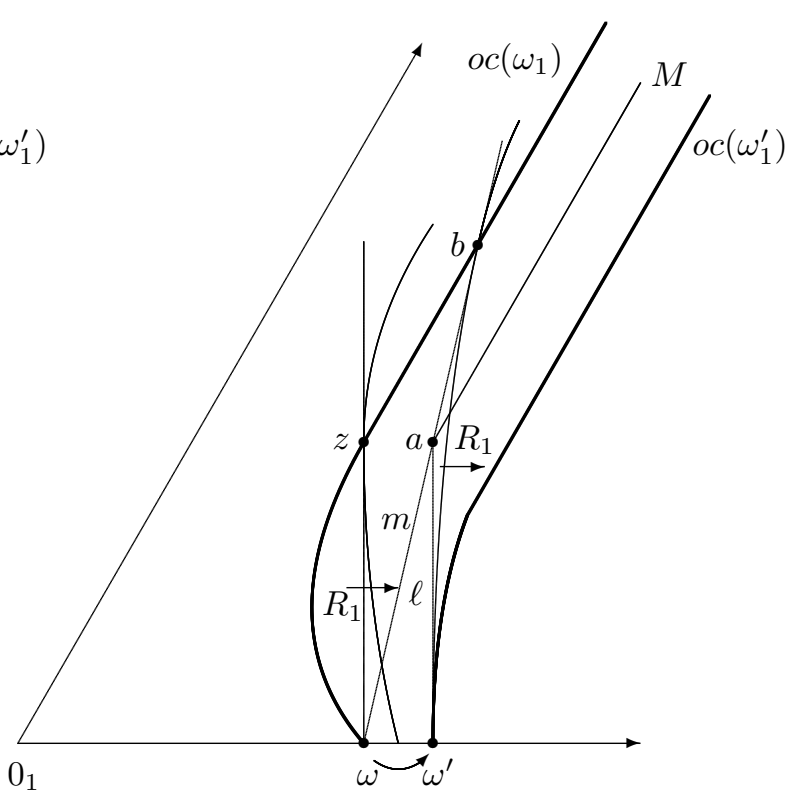

(b)

Figure 6: Generating agent 1's map for Example 1.

the same, so his offer curve from $\omega_{2}$ is the symmetric image of $o c\left(\omega_{1}\right)$ with respect to the vertical line through $\omega$. The offer curves oc $\left(\omega_{1}\right)$ and $o c\left(\omega_{2}\right)$ intersect at $\omega$ and $z$. Thus, $\{z\}=L(R, \omega, Y)$.

Let $\beta>0$. Agent 1 borrows the amount $\beta$ of the private good. We enlarge the Kolm triangle so as to accommodate the increase in his endowment. Let $\omega^{\prime}$ be the new endowment profile. In the enlarged Kolm triangle, let $o c^{\prime}\left(\omega_{2}\right)$ be agent 2's offer curve redrawn from $\omega_{2}^{\prime}$. It is the translate of $o c\left(\omega_{2}\right)$ by $\operatorname{tr}\left(\omega, \omega^{\prime}\right)$. Let $z^{\prime}$ be a point of $o c^{\prime}\left(\omega_{2}\right)$ whose public good component is smaller than the public good component of $z$. Agent 1's intended offer curve from $\omega_{1}^{\prime}$, oc $\left(\omega_{1}^{\prime}\right)$, consists of a backward-bending arc from $\omega_{1}^{\prime}$ to $z^{\prime}$, also followed by a half-line parallel to the left side of the triangle. It admits a vertical tangency line at $\omega_{1}^{\prime}$. Our objective is to specify preferences for agent 1 rationalizing $o c\left(\omega_{1}\right)$ as his offer curve from $\omega_{1}$ and $o c\left(\omega_{1}^{\prime}\right)$ as his offer curve from $\omega_{1}^{\prime}$.

We show in Figure 6 how to construct agent 1's indifference curve through $\omega_{1}^{\prime}$ as well as all of his higher indifference curves. Let $a$ be obtained from $z$ by $\operatorname{tr}\left(\omega, \omega^{\prime}\right)$ and $M$ be the half-line emanating from $a$ that is parallel to the left side of the triangle. Let $\bar{\ell}$ be any line emanating from $\omega^{\prime}$ whose slope is positive and greater than the slope of the left side of the triangle (Figure 6a). We will associate with it a point of $o c\left(\omega_{1}\right)$ and a point of $o c\left(\omega_{1}^{\prime}\right)$. Let $\bar{z}$ be the point of intersection of $\bar{\ell}$ with $o c\left(\omega_{1}^{\prime}\right)$. The line $\bar{\ell}$ intersects $M$ at $\bar{a}$. Next, we draw the line $\bar{m}$ from $\omega$ passing through $\bar{a}$. This line intersects oc $\left(\omega_{1}\right)$ at $\bar{b}$. We now draw an indifference curve for agent 1 that is tangent to $\bar{\ell}$ at $\bar{z}$ and to $\bar{m}$ at $\bar{b}$. We have rationalized $\bar{z}$ as a point of $o c\left(\omega_{1}^{\prime}\right)$ and $\bar{b}$ as a point 
of $o c\left(\omega_{1}\right)$.

As $\bar{\ell}$ becomes steeper and steeper, the point $\bar{z}$ gets closer and closer to $\omega^{\prime}$. At the limit, $\bar{\ell}$ is vertical and $\bar{z}=\omega^{\prime}$ (Figure $6 \mathrm{~b}$ ). Let us refer to $\bar{\ell}$ when it assumes this vertical position as $\ell$. Then, it intersects $M$ at $a$ and the line $m$ from $\omega$ passing through $a$ intersects oc $\left(\omega_{1}\right)$ at $b$. Now, we draw an indifference curve for agent 1 that is tangent to $\ell$ at $\omega_{1}^{\prime}$ and tangent to $m$ at $b$. This is the left-most indifference curve that we generate for agent 1 in this manner. We have rationalized $\omega^{\prime}$ as a point of $o c\left(\omega_{1}^{\prime}\right)$ and $b$ as a point of $o c\left(\omega_{1}\right)$.

All of these indifference curves can be drawn without crossing, and we have now generated all of agent 1's indifference curves to the right of the indifference curve through $\omega^{\prime}$, and in the process rationalized the part of $o c\left(\omega_{1}\right)$ that lies above $b$ as well as the whole of $o c\left(\omega_{1}^{\prime}\right)$. Note that because $z^{\prime}$ is to the right of $\ell$, its translate by $-\operatorname{tr}\left(\omega, \omega^{\prime}\right)$ is to the right of the vertical line through $\omega$. It is now easy to complete $R_{1}$ to the left of the indifference curve through $\omega^{\prime}$ that we just specified for him so as to rationalize the remainder of $o c\left(\omega_{1}\right)$ (the arc from $\omega$ to $z$ and the segment $[z, b]$ ), and so that the indifference curve through $z$ passes to the left of the translate of $z^{\prime}$ by $-\operatorname{tr}\left(\omega, \omega^{\prime}\right)$. Then, agent 1 prefers his final bundle, $\tilde{z}_{1} \equiv z_{1}^{\prime}-(\beta, 0)$, to $z_{1}$. He is made better off through borrowing.

Note that because none of the offer curves involved in the construction bends back down, in each of the two economies we consider, the economy with endowment profile $\omega$ and the economy with endowment profile $\left(\omega_{1}^{\prime}, \omega_{2}\right)$, there is a unique Lindahl allocation.

Remark 1 In the construction of the example, we fix $\omega$ and then specify $\beta$ to be an arbitrary positive number. Thus, the example shows that no matter how small the amount is that agent 1 is allowed to borrow in relation to his endowment, his preferences may be such that he can benefit from doing so.

\section{Borrowing versus withholding}

Another form of manipulation has been considered in the literature. It involves withholding of some of one's endowment for private consumption later. A rule is withholding-proof if no agent ever benefits from such behavior.

Withholding-proofness: For each $e \equiv(R, \omega, Y) \in \mathcal{E}^{N}$, each $i \in N$, and each $\omega^{\prime} \in \mathbb{R}_{+}^{N} \times \mathbb{R}_{+}$, if $\omega_{i x}^{\prime}<\omega_{i x}, \omega_{-i x}^{\prime}=\omega_{-i x}$, and $\omega_{y}^{\prime}=0$, then it is not 


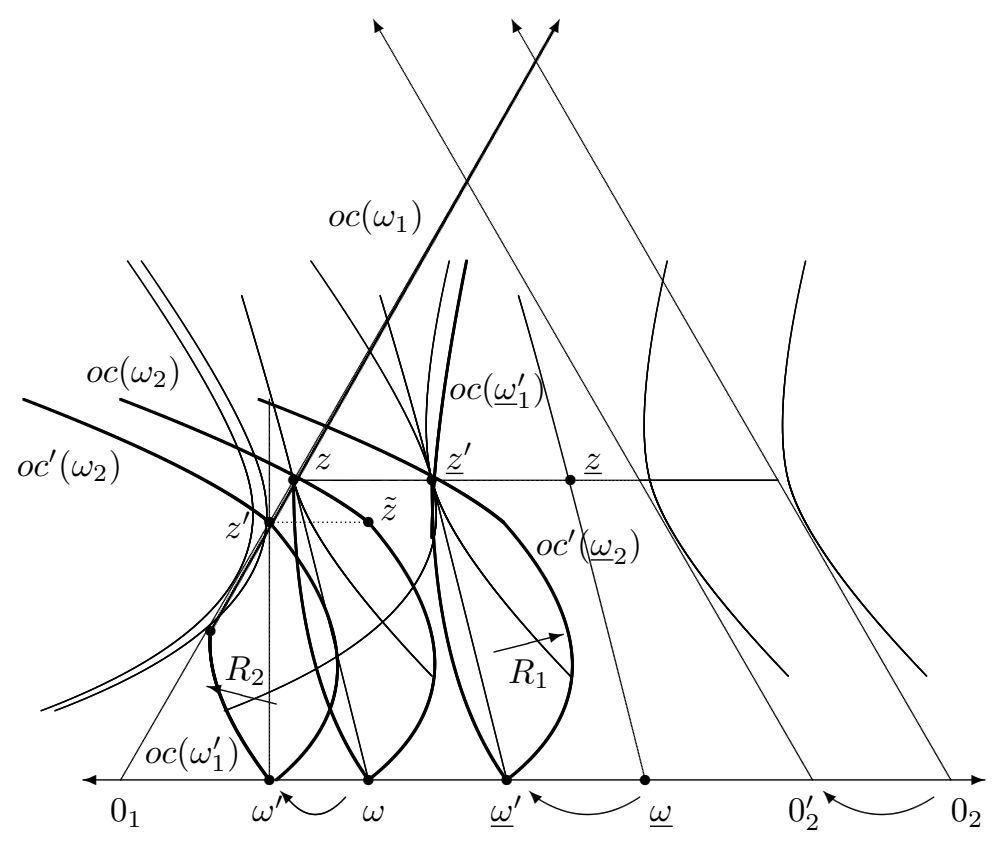

Figure 7: On the strictly convex, smooth, and quasi-linear domain, the Lindahl rule is not withholding-proof (Example 2).

the case that for some $z \in \varphi(R, \omega, Y)$ and some $z^{\prime} \in \varphi\left(R, \omega^{\prime}, Y\right),\left[z_{i}^{\prime}+\left(\omega_{i x}-\right.\right.$ $\left.\left.\omega_{i x}^{\prime}, 0\right)\right] P_{i} z_{i}$.

It is known that the Lindahl rule is not withholding-proof on the classical domain (Thomson, 1979, 1999). The examples described next show that this negative result still holds on either the strictly convex, smooth, and quasilinear domain, or on the strictly convex and homothetic domain. Thus, in the light of Section 3, it appears that open-economy borrowing-proofness is satisfied more generally than withholding-proofness.

Example 2 On the domain of strictly convex, smooth, and quasi-linear preferences, the Lindahl rule is not withholding-proof. The proof is by means of a strictly convex and smooth economy $(R, \omega, Y) \in \mathcal{E}_{q l}^{N}$, where $N \equiv\{1,2\}$, illustrated in Figure 7. Let $z \equiv L(R, \omega, Y)$. Agent 1 withholds the amount $\beta$ of the private good. Let $\omega^{\prime}$ be the new endowment profile in the reduced Kolm triangle. Let $z^{\prime} \equiv L\left(R, \omega^{\prime}, Y\right)$. This allocation is the intersection of $o c\left(\omega_{1}^{\prime}\right)$, which is truncated, and $o c^{\prime}\left(\omega_{2}\right)$. The price faced by agent 1 for the public good at equilibrium has turned in his favor. After adding to $z_{1}^{\prime}$ the vector agent 1 withheld, we obtain $z_{1}^{\prime}+(\beta, 0)$. To show a violation of withholdingproofness, it now suffices to draw agent 1's indifference curve through $z_{1}$ sufficiently close to the line passing through $\omega$ and $z$ to ensure that agent 1 prefers $z_{1}^{\prime}+(\beta, 0)$ to $z_{1}$. Then, he benefits from withholding.

If we had started from the endowment profile $\underline{\omega}$, for which the resulting Lindahl allocation is interior, then for a range of values of the amount with- 


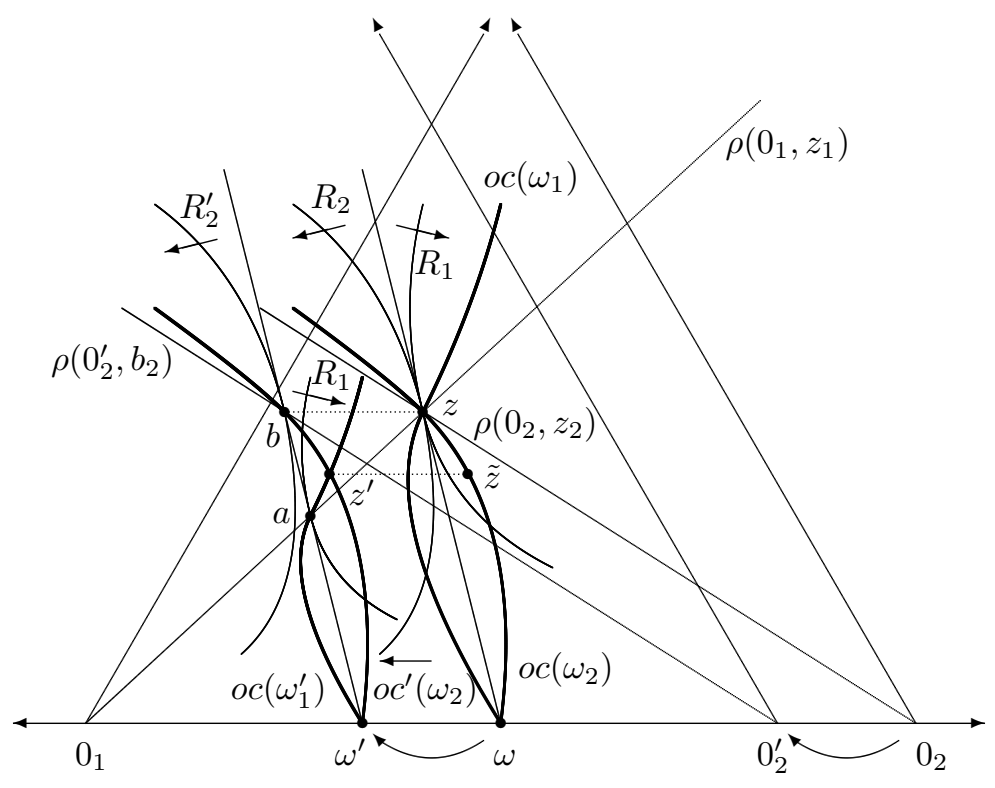

Figure 8: On the strictly convex and homothetic domain, the Lindahl rule is not withholding-proof (Example 3).

held, the new endowment profile would have resulted in an interior Lindahl allocation. In the figure, an endowment profile that achieves this is $\underline{\omega}^{\prime}$. Then the Lindahl allocation from this new endowment, $\underline{z}^{\prime}$ on the figure, would be obtained from the Lindahl allocation from $\underline{\omega}$ by $\operatorname{tr}\left(\underline{\omega}, \underline{\omega}^{\prime}\right)$, and agent 1 would not have benefitted from withholding.

Example 3 On the domain of strictly convex and homothetic preferences, the Lindahl rule is not withholding-proof. The proof is by means of a strictly convex economy $(R, \omega, Y) \in \mathcal{E}_{\text {hom }}^{N}$, where $N \equiv\{1,2\}$, illustrated in Figure 8 . Let $z \equiv L(R, \omega, Y)$. Agent 1 withholds the amount $\beta$ of the private good. Let $\omega^{\prime}$ be the new endowment profile in the reduced Kolm triangle and $z^{\prime} \equiv$ $L\left(R, \omega^{\prime}, Y\right)$. The essential characteristic of agent 1's offer curve from his new endowment, $o c\left(\omega_{1}^{\prime}\right)$, is that on each price line that he may face, agent 1's most preferred trade is smaller than it was from his initial endowment. Agent 2's offer curve measured from the new origin $0_{2}^{\prime}$ in the reduced triangle, oc $\left(\omega_{2}\right)$, is obtained by subjecting his initial offer curve $o c\left(\omega_{2}\right)$ to $\operatorname{tr}\left(\omega, \omega^{\prime}\right)$. The two offer curves from $\omega^{\prime}$ cross to the right of the line obtained by subjecting the initial equilibrium price line to $\operatorname{tr}\left(\omega, \omega^{\prime}\right)$. Thus, $z_{1}^{\prime}+(\beta, 0)$ is to the right of the initial equilibrium price line. It now suffices to draw agent 1 's indifference curve through $z_{1}$ sufficiently close to the line passing through $\omega$ and $z$ to ensure that agent 1 benefits from withholding.

Remark 2 A parallel observation to one made in connection to Example 1 
holds: Examples 2 and 3 show that no matter how small the amount is that agent 1 withholds in relation to his endowment, his preferences may be such that he could benefit from doing so.

Both borrowing and withholding have the effect of "bringing the attention of the rule" to a different part of the manipulating agent's consumption space. An examination of the proofs of our results concerning the two behaviors reveals that the difference in the conclusions comes from the fact that for the classes of preferences on which we have focused, quasi-linear preferences and homothetic preferences, augmenting one's endowment through borrowing and decreasing it through withholding have predictably opposite effects on offer curves; in the borrowing case, demand for the public good increases at every price, and in the withholding case, demand decreases at every price. Because the other agent has a well-behaved map, this leads to a predictably more favorable individualized price for the manipulating agent in the case of withholding, and a predictably less favorable individualized price for him in the case of borrowing.

\section{Closed-economy borrowing-proofness}

Let us now imagine that an agent borrows from some other agent present in the economy, not from outside sources; after the rule is applied, and the agent has returned what he borrowed, he may be better off, but in order to guarantee the participation of the agent from whom he borrowed, this agent should end up at least as well off as he would have been otherwise. (Of course, the borrower could borrow from several agents. Then each of them should end up at least as well off as if he had not borrowed.) The property of immunity to behavior of this kind is formulated in Thomson (2005) under the name of "closed-economy borrowing-proofness". For a selection from the Pareto correspondence, this property is automatically met if there are only two agents, so we need to consider economies with a least three agents, and the Kolm triangle is not available anymore. Formally, the requirement is the following:

Closed-economy borrowing-proofness: For each $e \equiv(R, \omega, Y) \in \mathcal{E}^{N}$, each $\{i, j\} \subseteq N$, and each $\omega^{\prime} \in \mathbb{R}_{+}^{N} \times \mathbb{R}_{+}$, if $\omega_{i x}^{\prime}>\omega_{i x}, \omega_{i x}^{\prime}+\omega_{j x}^{\prime}=\omega_{i x}+\omega_{j x}$, for each $k \in N \backslash\{i, j\}, \omega_{k x}^{\prime}=\omega_{k x}$, and $\omega_{y}^{\prime}=0$, then it is not the case that for some 
$z \in \varphi(R, \omega, Y)$ and some $z^{\prime} \in \varphi\left(R, \omega^{\prime}, Y\right)$, we have $\left[z_{i}^{\prime}-\left(\omega_{i x}^{\prime}-\omega_{i x}, 0\right)\right] P_{i} z_{i}$ and $\left[z_{j}^{\prime}+\left(\omega_{i x}^{\prime}-\omega_{i x}, 0\right)\right] R_{j} z_{j}$.

We start with two positive results.

Proposition 3 On the domain of strictly convex, smooth, and quasi-linear preferences, the Lindahl rule is closed-economy borrowing-proof.

Proof: Consider a strictly convex and smooth economy $(R, \omega, Y) \in \mathcal{E}_{q l}^{N}$, and let $z \in L(R, \omega, Y)$. Let $\{i, j\} \subseteq N$ and suppose that agent $i$ borrows the amount $\beta$ of the private good from agent $j$. Let $\omega_{i x}^{\prime} \equiv \omega_{i x}+\beta, \omega_{j x}^{\prime} \equiv \omega_{j x}-\beta$, and for each $k \in N \backslash\{i, j\}, \omega_{k x}^{\prime} \equiv \omega_{k x}$, and $\omega_{y}^{\prime}=0$. Let $z^{\prime} \equiv L\left(R, \omega^{\prime}, Y\right)$. Agent $i$ 's final bundle is $z_{i}^{\prime}-(\beta, 0)$. For him to benefit, he should face a lower price for the public good (his budget line should become steeper). By quasi-linearity, this implies that $y^{\prime}>y$. For each $k \in N \backslash\{i\}$, if $z_{k}^{\prime}$ is a maximizing consumption bundle in agent $k$ 's budget set, the price he should face for the public good should be at most as large as it was initially. However it is incompatible with feasibility that every agent faces a lower price.

Proposition 4 On the domain of strictly convex and homothetic preferences, the Lindahl rule is closed-economy borrowing-proof.

Proof: The proof is the same as that of Proposition 3 until the sentence beginning with "By quasi-linearity" but the same conclusion is reached by invoking homotheticity. The proof concludes as does the previous one.

The proofs of the two previous propositions make it clear that what underlies them is, once again, normality of the public good. On the classical domain, this property is not necessarily met, and we have the following negative result.

Example 4 On the strictly convex, smooth, and classical domain, the Lindahl rule is not closed-economy borrowing-proof. Let $N \equiv\{1,2,3\}$. The proof is by means of an economy in $\mathcal{E}_{c l}^{N}$ with strictly convex and smooth preferences (Figures 9 and 10). The initial endowment profile is denoted $\omega \in \mathbb{R}_{+}^{N} \times \mathbb{R}_{+}$. Agent 1 borrows resources from agent 2, the resulting endowment profile being denoted $\omega^{\prime}$. The production technology is linear, each unit of input yielding one unit of output, and as before, the production set is denoted $Y$. The initial economy is $(R, \omega, Y)$ and after agent 1 borrows, it 


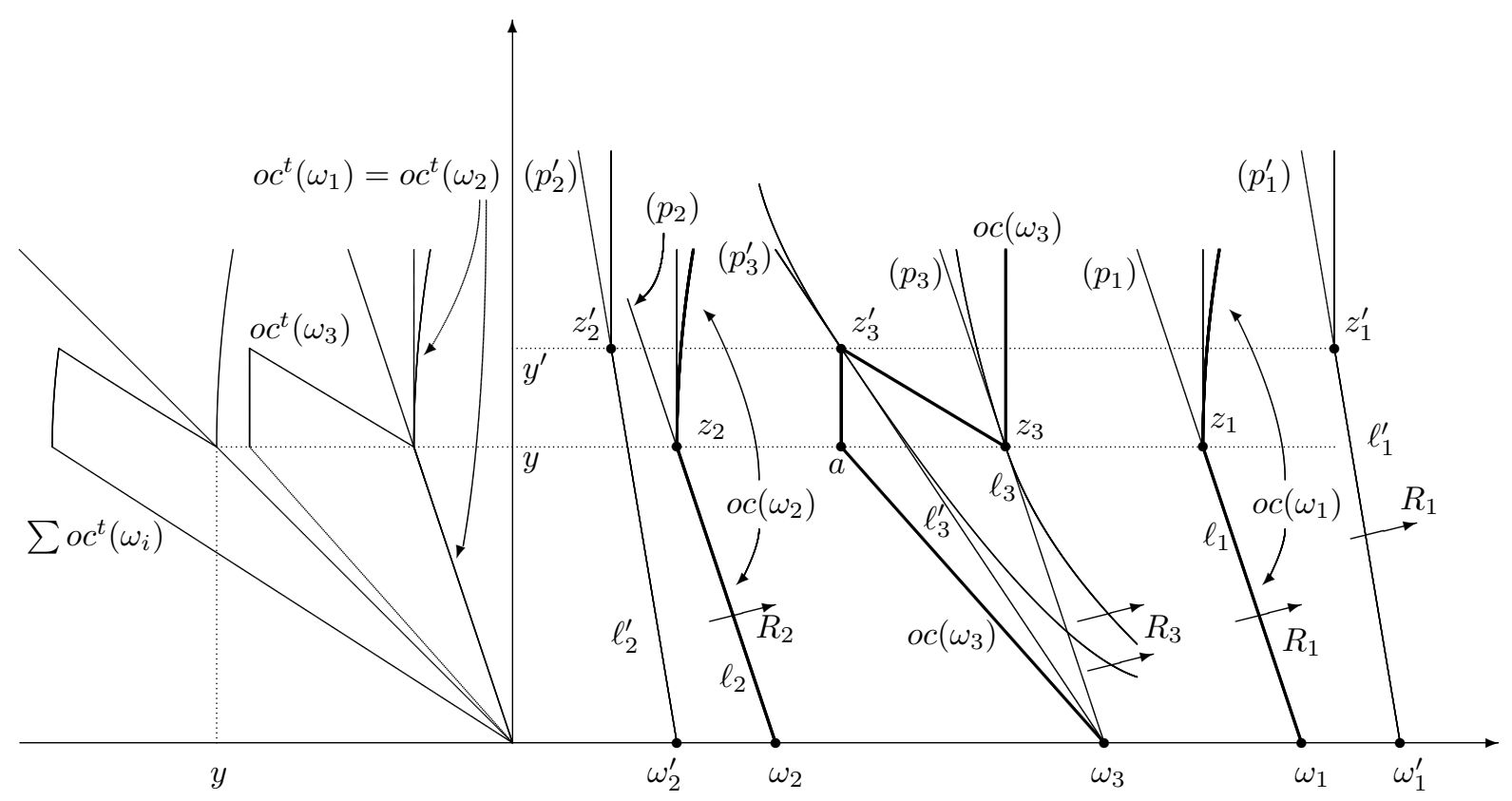

Figure 9: On the strictly convex, smooth, and classical domain, the Lindahl rule is not closed-economy borrowing-proof (Example 4).

is $\left(R, \omega^{\prime}, Y\right)$. There is a unique Lindahl allocation, both initially and after the change in endowments.

Let $\omega_{1 x}, \omega_{1 x}^{\prime}, \beta \in \mathbb{R}_{+}$be such that $\omega_{1 x}^{\prime}=\omega_{1 x}+\beta$. Let $y, y^{\prime}>0$ be such that $y<y^{\prime}$. Let $\omega_{2 x}, \omega_{2 x}^{\prime}>0$ be such that $\omega_{2 x}^{\prime}=\omega_{2 x}-\beta$. Let $p_{2}, p_{2}^{\prime} \in \mathbb{R}_{+}$be such that (i) $0<p_{2}^{\prime}<p_{2}<1$, (ii) the point $z_{2}^{\prime}$ of ordinate $y^{\prime}$ on the line $\ell_{2}^{\prime}$ emanating from $\omega_{2}^{\prime}$ and normal to $\left(1, p_{2}^{\prime}\right)$ has a positive abscissa, and (iii) the point $z_{2}$ of ordinate $y$ on the line $\ell_{2}$ emanating from $\omega_{2}$ and normal to $\left(1, p_{2}\right)$ is to the right of $\ell_{2}^{\prime}$. These conditions imply that $z_{2}$ has a positive abscissa.

Let $p_{1}, p_{1}^{\prime} \in \mathbb{R}_{+}$be such that (i) $0<p_{1}^{\prime}<p_{1}$ and $p_{3} \equiv 1-p_{1}-p_{2}<1$ and (ii) the point $z_{1}$ of ordinate $y$ on the line $\ell_{1}$ emanating from $\omega_{1}$ and normal to $\left(1, p_{1}\right)$ and the point $z_{1}^{\prime}$ of ordinate $y^{\prime}$ on the line $\ell_{1}^{\prime}$ emanating from $\omega_{1}^{\prime}$ and normal to $\left(1, p_{1}^{\prime}\right)$ have positive abscissas. Let $p_{3}^{\prime} \equiv 1-p_{1}^{\prime}-p_{2}^{\prime}$. Since $p_{1}^{\prime}<p_{1}$ and $p_{2}^{\prime}<p_{2}$, then $p_{3}^{\prime}>p_{3}$. Let $\omega_{3 x} \in \mathbb{R}_{+}$be such that the point $z_{3}^{\prime}$ of ordinate $y^{\prime}$ on the line $\ell_{3}^{\prime}$ emanating from $\omega_{3}$ and normal to $\left(1, p_{3}^{\prime}\right)$ has a positive abscissa. (The figures depict an economy for which $p_{1}=p_{2}=p_{3}$ and $p_{1}^{\prime}=p_{2}^{\prime}$.) Since $y<y^{\prime}$ and $p_{3}<p_{3}^{\prime}$, the point $z_{3}$ of ordinate $y$ on the line $\ell_{3}$ emanating from $\omega_{3}$ and normal to $\left(1, p_{3}\right)$ also has a positive abscissa. Next, we specify offer curves, at first not insisting on either strict convexity or smoothness of preferences.

Let $R_{1}$ be such that (i) the indifference curve through $\omega_{1}$ consists of $\operatorname{seg}\left[\omega_{1}, z_{1}\right]$ and the vertical half-line emanating from $z_{1}$, and (ii) the indifference curve through $\omega_{1}^{\prime}$ consists of $\operatorname{seg}\left[\omega_{1}^{\prime}, z_{1}^{\prime}\right]$ and the vertical half-line emanating from $z_{1}^{\prime}$. It follows from (i) that oc $\left(\omega_{1}\right)$ contains $\operatorname{seg}\left[\omega_{1}, z_{1}\right]$ and that above the horizontal line of ordinate $y$, it lies to the right of the half-line em- 


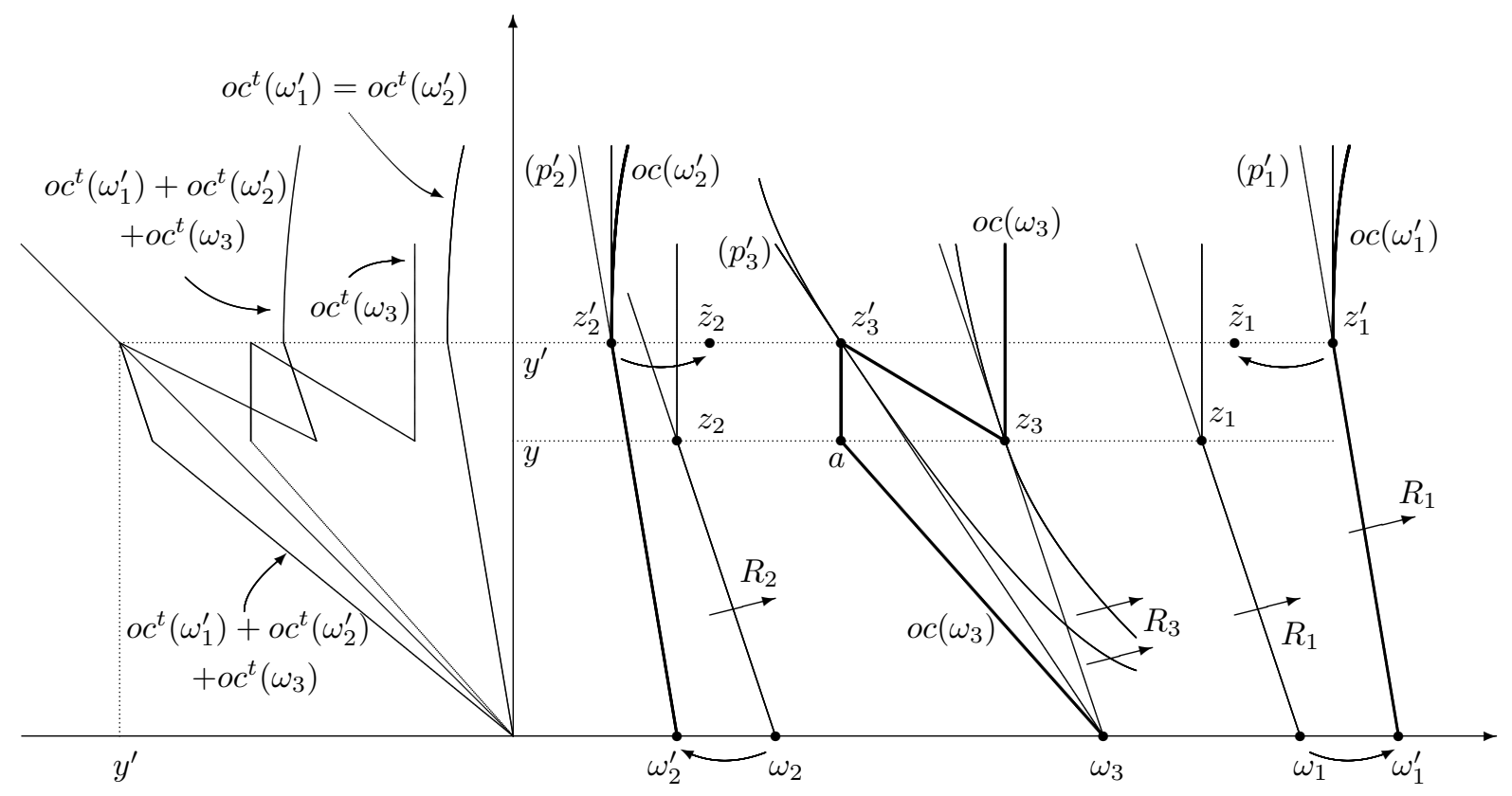

Figure 10: On the strictly convex, smooth, and classical domain, the Lindahl rule is not closed-economy borrowing-proof (Example 4 continued).

anating from $z_{1}$. Similarly, it follows from (ii) that oc $\left(\omega_{1}^{\prime}\right)$ contains $\operatorname{seg}\left[\omega_{1}^{\prime}, z_{1}^{\prime}\right]$ and that above the horizontal line of ordinate $y^{\prime}$, it lies to the right of the half-line emanating from $z_{1}^{\prime}$.

Let $R_{2}$ be such that (i) the indifference curve through $\omega_{2}$ consists of $\operatorname{seg}\left[\omega_{2}, z_{2}\right]$ and the vertical half-line emanating from $z_{2}$, and (ii) the indifference curve through $\omega_{2}^{\prime}$ consist of $\operatorname{seg}\left[\omega_{2}^{\prime}, z_{2}^{\prime}\right]$ and the vertical half-line emanating from $z_{2}^{\prime}$. It follows from (i) that oc $\left(\omega_{2}\right)$ contains $\operatorname{seg}\left[\omega_{2}, z_{2}\right]$ and that above the horizontal line of ordinate $y$, it lies to the right of the half-line emanating from $z_{2}$. Similarly, it follows from (ii) that oc $\left(\omega_{2}^{\prime}\right)$ contains $\operatorname{seg}\left[\omega_{2}^{\prime}, z_{2}^{\prime}\right]$ and that above the horizontal line of ordinate $y^{\prime}$, it lies to the right of the half-line emanating from $z_{2}^{\prime}$.

Let $a \equiv\left(a_{1}, a_{2}\right) \in \mathbb{R}_{+}^{2}$ be such that $a_{1}=x_{3}^{\prime}$ and $a_{2}<y^{\prime}\left(\right.$ say $\left.a_{2}=y\right)$. Let $R_{3}$ be such that $o c_{3}\left(\omega_{3}\right)$ consists of bro.seg $\left[\omega_{3}, a, z_{3}^{\prime}, z_{3}\right]$, followed by the vertical half-line emanating from $z_{3}$.

Let $\omega_{x} \equiv\left(\omega_{1 x}, \omega_{2 x}, \omega_{3 x}\right), \omega_{3 x}^{\prime}=\omega_{3 x}$, and $\omega_{x}^{\prime} \equiv\left(\omega_{1 x}^{\prime}, \omega_{2 x}^{\prime}, \omega_{3 x}^{\prime}\right)$. Let $z \equiv$ $\left(x_{1}, x_{2}, x_{3}, y\right)$ and $z^{\prime} \equiv\left(x_{1}^{\prime}, x_{2}^{\prime}, x_{3}^{\prime}, y^{\prime}\right)$. We have $z \in L(R, \omega, Y)$ and $z^{\prime} \in$ $L\left(R, \omega^{\prime}, Y\right)$. We claim that in fact, $z$ and $z^{\prime}$ are the only Lindahl allocations for $\omega$ and $\omega^{\prime}$ respectively. For each $\tilde{y} \in \mathbb{R}_{+}$, we identify the individualized prices for the public good at which each of the three agents would demand this level of the public good. From these, we deduce the total amount collected 
if the public good is offered at level $\tilde{y}$. For a Lindahl equilibrium, this total should be equal to the cost of producing the public good at that level. Given our assumptions on the technology, this cost is also $\tilde{y}$. Geometrically, it is convenient to translate the three offer curves horizontally to the origin, add them up horizontally, then check if the resulting aggregate demand curve and the supply curve, which is simply the line of slope -1 emanating from the origin, cross at a point of ordinate $\tilde{y}$. If this is the only point of intersection, we have a unique Lindahl allocation. In Figures 9 and 10, the translated offer curves of the initial economy are denoted $o c^{t}\left(\omega_{1}\right)$, oc ${ }^{t}\left(\omega_{2}\right)$, and $o c^{t}\left(\omega_{3}\right)$. Their aggregate is denoted $\sum o c^{t}\left(\omega_{i}\right)$. After agent 1 borrows from agent 2, these two agents' translated offer curves are denoted $o c^{t}\left(\omega_{1}^{\prime}\right)$ and $o c^{t}\left(\omega_{2}^{\prime}\right)$. The aggregate translated offer curve is then written as $o c^{t}\left(\omega_{1}^{\prime}\right)+o c^{t}\left(\omega_{2}^{\prime}\right)+o c^{t}\left(\omega_{3}\right)$.

In Figures 9 and 10, uniqueness of the points of intersection of the aggregate demand curve and the supply curve can be verified.

The Lindahl allocation $z^{\prime}$ for the endowment profile $\omega^{\prime}$ is such that, defining $\tilde{z} \equiv\left(z_{1}^{\prime}-(\beta, 0), z_{2}^{\prime}+(\beta, 0)\right)$, we have $\tilde{z}_{1} P_{1} z_{1}$ and $\tilde{z}_{2} R_{2} z_{2}$. To conclude the proof, it suffices to take strictly convex and smooth approximations to the preferences just defined. This can easily be done but once again, we dispense with analytical expressions. ${ }^{9}$

Remark 3 Here too, a similar observation to Remark 1 made in connection to Example 1 holds: Example 4 can be specified, and we have constructed it with that objective in mind, to show that no matter how small the amount that agent 1 is allowed to borrow in relation to his endowment and in relation to the endowment of the lender, (we may require for instance that the ratio of the amount borrowed over either of these endowments not exceed a certain value,) there are economies such that he could benefit from borrowing. When introducing $\omega_{1 x}$ and $\beta$, it suffices to add the requirement that $\frac{\beta}{\omega_{1 x}}$ be no greater than the desired value; then, in introducing $\omega_{2 x}$, to add the requirement that $\frac{\beta}{\omega_{2 x}}$ also be no greater than the desired value.

\section{Conclusion}

We close by relating our study to existing literature and stating open questions. The present paper is indeed only a first step in the study of the opportunity through manipulation of endowments that we envision.

\footnotetext{
${ }^{9}$ The preferences of both agents 1 and 2 can in addition be specified to be homothetic.
} 
1. More than one private good. In a related paper in which we examine borrowing-proofness in the context of classical private good economies (Thomson, 2009), we obtain a number of negative results concerning the compatibility of this requirement with efficiency and one or the other of several distributional requirements. We prove these results for economies with two goods. Such conclusions can easily be extended to economies in which public goods are also present. It suffices to specify preferences so that, at all efficient allocations, the public goods are not produced because the demand does not justify the expense. Thus, we have limited ourselves here to economies with only one private good. Since the public goods themselves cannot be borrowed, manipulation opportunities through borrowing are more limited in the present context than they are in economies with only private goods. Our results are somewhat more positive than in such economies, as we have found a number of natural preference domains on which the Lindahl solution is immune to manipulation through borrowing.

2. Other rules. In a companion paper (Thomson, 2010), we study the borrowing-proofness of other allocation rules in the context of the same model. These rules are inspired by the literature on fairness.

3. Introducing a cost of borrowing. To be able to borrow, an agent should in general pay a fee. ${ }^{10}$ The higher the fee, the less likely will borrowing be worthwhile. Thus, it would be interesting to obtain results describing the extent to which introducing a cost of borrowing will prevent the sort of manipulation with which we are concerned. However, results independent of the preference profile and of the particular initial endowment profile are unlikely.

4. Manipulation involving groups of agents. Other, more sophisticated, forms of borrowing than the one we have analyzed can be defined, involving groups of agents.

First, several agents may coordinate their borrowing and, after each of them has returned what he borrowed, each of them end up with a bundle they he prefers.

Second, their opportunities to gain may be enhanced if, instead of each of them returning the very bundle that he borrowed, they jointly return what they borrowed in total.

\footnotetext{
${ }^{10}$ We do not want to use the expression "interest rate" because, as we noted earlier, our model is not an intertemporal one.
} 
Third, they may obtain additional improvements in their welfares if, after returning what they borrowed individually or a group, they carry out transfers among themselves.

Fourth, in a closed economy, as long as a lender's welfare ends up at least as high at what it would have been if he did not lent, he should be willing to lend; thus, one could specify the obligation of the borrowers to simply return a bundle that allows the lender to achieve the welfare he would achieve if he had not lent. The idea can be applied to borrowing by a group from some other group.

We leave the analysis of these behaviors to future study, limiting ourselves to noting that for each of the negative results we have obtained by focusing on manipulation by one agent only, the situation is of course worsened when agents may coordinate their manipulation.

5. Borrowing games. Once it is known that a rule is not immune to manipulation, one may want to understand what happens when all agents attempt separately to manipulate to their own advantage. Full-fledged game theoretic analysis of a rule requires that a manipulation game be associated with the rule, and its equilibrium allocations identified and compared to the allocations the rule would recommend under truthful behavior. Local conditions for a list of strategies to be an equilibrium of the game of endowment misrepresentation associated with the Lindahl rule are derived by Thomson (1979) who also calculates simple examples. The question is addressed in the case of economies with normal goods by Sertel and Sanver (1999).

6. Other classes of problems. On the basis of this paper and Thomson (2005), some understanding of the strength of borrowing-proofness is emerging. A more definitive assessment will have to wait until other classes of problems are studied. Economies with indivisible goods, both when these are the only goods available and when in addition, there is an infinitely divisible good that can be used to perform compensations, are investigated by Atlamaz and Thomson (2007), who report a combination of positive and negative results. The withholding issue is considered for economies with indivisible goods by Atlamaz and Klaus (2007). 


\section{REFERENCES}

Atlamaz, M. and B. Klaus, "Manipulation via endowments in exchange markets with indivisible goods", Social Choice and Welfare 28 (2007), 1-18.

Atlamaz, M. and W. Thomson, "Borrowing-proofness in economies with indivisible goods", mimeo, 2007.

Aumann, R. and B. Peleg, "A note on Gale's example," Journal of Mathematical Economics 1 (1974), 209-211.

Ching, S. and L. Zhou, "Multi-valued strategy-proof social choice rules", Social Choice and Welfare 19 (2002), 569-580.

Dolbear, F.T., "On the theory of optimal externality", American Economic Review 57 (1967), 90-103.

Gale, D., "Exchange equilibrium and coalitions: an example", Journal of Mathematical Economics 1 (1974), 63-66.

Kolm, S.C., La Valeur Publique, Paris: Dunod, 1971.

Lindahl, E., Die Gerechtichkeit der Besteurung, Lund: Gleerup 1919, reprinted in part as "Just taxation - a positive solution" in Classics in the Theory of Public Finance, edited by R.A. Musgrave and A.T. Peacok. London: Macmillan, 1958, 168-176.

Postlewaite, A., "Manipulation via endowments", Review of Economic Studies 46 (1979), 255-262.

Sertel, M., "Manipulating Lindahl equilibrium via endowments", Economics Letters 46 (1994), 167-171.

Sertel, M. and R. Sanver., "Equilibrium outcomes of Lindahl-endowment pretension games", European Journal of Political Economy 15 (1999), 149-162.

Thomson, W., "The equilibrium allocations of Walras and Lindahl manipulation games," University of Minnesota, Center for Economic Research Discussion Paper No. 79-111, July 1979.

__ , "Monotonic allocation rules," University of Rochester Discussion Paper No. 116, December 1987a, revised 1995.

—_, "Monotonic allocation rules in economies with public goods," University of Rochester Discussion Paper No. 117, December 1987b, revised 1995.

—_, "Economies with public goods: an elementary geometric exposition", Journal of Public Economic Theory 1 (1999), 139-176.

—_, "New variable-population paradoxes for resource allocation", mimeo, University of Rochester, 2007. 
_ _ "Borrowing-proofness", University of Rochester RCER working paper, 2009.

_, "Borrowing-proofness in economies with public goods", 2010. 